\title{
Bioelectrical and cytoskeletal patterns correlate with altered axial polarity in the follicular epithelium of the Drosophila mutant gurken
}

\author{
Susanne Katharina Schotthöfer and Johannes Bohrmann*
}

\begin{abstract}
Background: Bioelectrical signals are known to be involved in the generation of cell and tissue polarity as well as in cytoskeletal dynamics. The epithelium of Drosophila ovarian follicles is a suitable model system for studying connections between electrochemical gradients, patterns of cytoskeletal elements and axial polarity. By interactions between soma and germline cells, the transforming growth factor-a homolog Gurken (Grk) establishes both the anteroposterior and the dorsoventral axis during oogenesis.

Results: In the follicular epithelium of the wild-type (wt) and the polarity mutant grk, we analysed stage-specific gradients of membrane potentials $\left(\mathrm{V}_{\text {mem }}\right)$ and intracellular $\mathrm{pH}\left(\mathrm{pH}_{\mathrm{i}}\right)$ using the potentiometric dye $\operatorname{DiBAC}_{4}(3)$ and the fluorescent pH-indicator 5-CFDA,AM, respectively. In addition, we compared the cytoskeletal organisation in the follicular epithelium of wt and grk using fluorescent phalloidin and an antibody against acetylated a-tubulin.

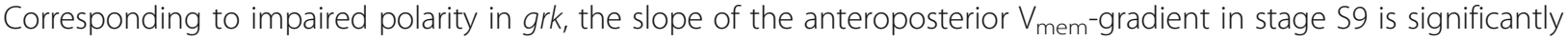
reduced compared to wt. Even more striking differences in $\mathrm{V}_{\mathrm{mem}}$ - and $\mathrm{pH}_{\mathrm{i}}$-patterns become obvious during stage S10B, when the respective dorsoventral gradients are established in wt but not in grk. Concurrent with bioelectrical differences, wt and grk exhibit differences concerning cytoskeletal patterns in the follicular epithelium. During all vitellogenic stages, basal microfilaments in grk are characterised by transversal alignment, while wt-typical condensations in centripetal follicle cells (S9) and in dorsal centripetal follicle cells (S10B) are absent. Moreover, in grk, longitudinal alignment of microtubules occurs throughout vitellogenesis in all follicle cells, whereas in wt, microtubules in mainbody and posterior follicle cells exhibit a more cell-autonomous organisation. Therefore, in contrast to wt, the follicular epithelium in grk is characterised by missing or shallower electrochemical gradients and by more coordinated transcellular cytoskeletal patterns.
\end{abstract}

Conclusions: Our results show that bioelectrical polarity and cytoskeletal polarity are closely linked to axial polarity in both wt and grk. When primary polarity signals are altered, both bioelectrical and cytoskeletal patterns in the follicular epithelium change. We propose that not only cell-specific levels of $\mathrm{V}_{\text {mem }}$ and $\mathrm{pH}_{\mathrm{i}}$, or the polarities of transcellular electrochemical gradients, but also the slopes of these gradients are crucial for cytoskeletal modifications and, thus, for proper development of epithelial polarity.

Keywords: Drosophila melanogaster, Oogenesis, Electrochemical gradient, Follicle cell, Gurken, Pattern formation

\footnotetext{
* Correspondence: bohrmann@bio2.rwth-aachen.de

RWTH Aachen University, Institut für Biologie II, Abt. Zoologie und

Humanbiologie, Worringerweg 3, 52056 Aachen, Germany
}

(c) The Author(s). 2020 Open Access This article is licensed under a Creative Commons Attribution 4.0 International License, which permits use, sharing, adaptation, distribution and reproduction in any medium or format, as long as you give appropriate credit to the original author(s) and the source, provide a link to the Creative Commons licence, and indicate if changes were made. The images or other third party material in this article are included in the article's Creative Commons licence, unless indicated otherwise in a credit line to the material. If material is not included in the article's Creative Commons licence and your intended use is not permitted by statutory regulation or exceeds the permitted use, you will need to obtain permission directly from the copyright holder. To view a copy of this licence, visit http://creativecommons.org/licenses/by/4.0/ The Creative Commons Public Domain Dedication waiver (http://creativecommons.org/publicdomain/zero/1.0/) applies to the data made available in this article, unless otherwise stated in a credit line to the data. 


\section{Background}

Spatiotemporal electrochemical patterns affect cytoskeletal dynamics and play a role in defining spatial coordinates of tissues and organs in several species [1-8]. Therefore, it is tempting to investigate $\mathrm{V}_{\mathrm{mem}^{-}}$and $\mathrm{pH}_{\mathrm{i}}{ }^{-}$ gradients in relation to cytoskeletal patterns in a Drosophila mutant with disturbed axial polarity. Ovarian follicles of the mutant gurken (grk) show morphological defects concerning both the anteroposterior (a-p) and the dorsoventral $(\mathrm{d}-\mathrm{v})$ axis $[9,10]$. In $g r k$ follicles, the oocyte nucleus $(\mathrm{ON})$ is located at the posterior end of the oocyte (Ooc), and the follicular epithelium (FE) has a transversally uniform appearance (Fig. 1). Since ON movement to an anterodorsal position fails to occur in grk, both the longitudinal and the transversal axis are not correctly defined [11, 12]. In addition, Grk is required for border-cell $(\mathrm{BC})$ migration to a position adjacent to the ON [13].

Grk, a transforming growth factor- $\alpha$ (TGF- $\alpha$ ) homo$\log$, is a ligand of the epidermal growth-factor receptor (EGFR) Torpedo (Top)/DER, and functions as a spatially restricted signal to activate the Egfr-pathway in follicle cells (FC) $[14,15]$. Two rounds of Grk-Egfr signalling at different times during oogenesis generate axial polarity. In early oogenesis (stages S6-7), Egfr-activation in posterior $\mathrm{FC}(\mathrm{pFC})$ defines a-p polarity, whereas in midoogenesis (S9), restriction of Egfr-activity to dorsal FC determines d-v polarity [16]. Localised Grk-Egfr signalling depends on the position of the ON $[9,17,18]$. In strong grk mutants, both anterior and posterior FC adopt anterior fates, as indicated by the anterior-specific FC-marker slbo, and micropylar structures develop at a

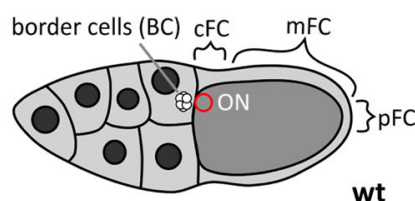

b

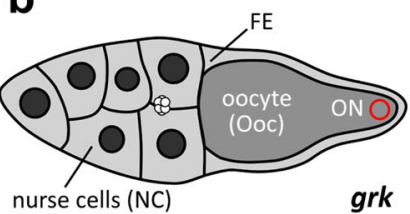

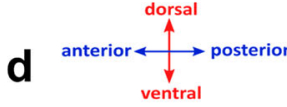

focal plane bMF/MT

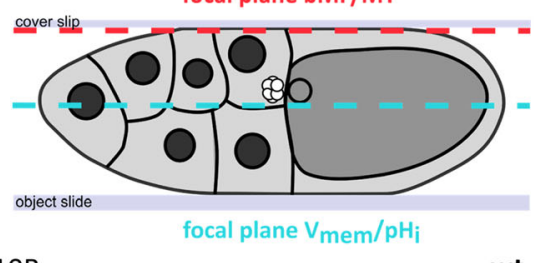

stage S10B
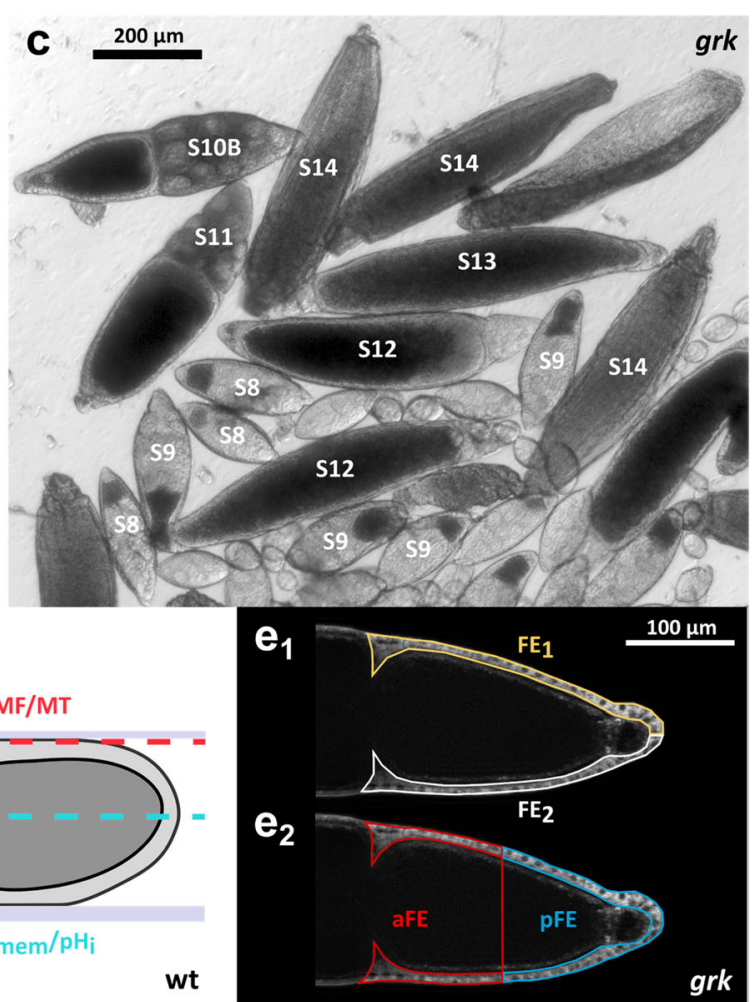

Fig. 1 Comparison of wt and grk follicles. a The dorsal side of wt S10B is defined by a thicker, columnar follicular epithelium (FE) and by an anterodorsal position of the oocyte nucleus (ON, red circle; $C F C$, centripetal follicle cells; mFC, mainbody follicle cells; pFC, posterior follicle cells). b grk S10B lacks dorsoventral ( $d-v)$ polarity and is characterised by a uniform cuboidal, ventralised FE covering the oocyte (Ooc). While, in wt $\mathrm{S} 10 \mathrm{~B}$, border cells $(\mathrm{BC})$ are located close to the $\mathrm{ON}$, in grk S10B, disrupted body-axis formation leads to undefined positioning of $\mathrm{BC}$ amongst the nurse cells (NC). The grk ON is often located at the posterior end of the Ooc in a typical protrusion. c Transheterozygous combinations of grk alleles HF48 and 2B6 result in ventralised grk follicles of all vitellogenic stages (S8-14; bright-field image). In S12-14, wt-typical dorsal respiratory appendages are missing and a second micropylar structure appears at the posterior end. $\mathbf{d}$ To visualise basal microfilaments (bMF) and microtubules (MT) in the FE, tangential optical sections using structured-illumination microscopy (SIM; focal plane: red line) were used. For

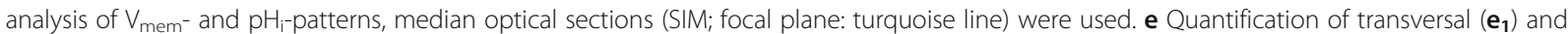
anteroposterior (a-p; $\mathbf{e}_{2}$ ) gradients of $\mathrm{V}_{\text {mem }}$ and $\mathrm{pH}_{\mathrm{i}}$, respectively, in the FE of S10B. Example of a grk follicle (SIM) where DiBAC-fluorescence intensities of $\mathrm{FE}_{1}$ (area marked in yellow) and $\mathrm{FE}_{2}$ (white) as well as of aFE (red) and pFE (blue) were measured using ImageJ ("mean grey value"). In wt follicles, the $d-v$ axis was identified via the anterodorsal position of the $O N$, and the fluorescence intensities of the dorsal and ventral FE were quantified accordingly 
both poles $[9,17]$. In the wild-type (wt), the $\mathrm{pFC}$, in return, signal back to the Ooc, inducing a reorganisation of the microtubule (MT) cytoskeleton and leading to the MT-dependent migration of the $\mathrm{ON}$ to an anterior position [12, 19]. As a result, the overlying FC receive the Grk-signal and adopt dorsal fates [16, 20, 21]. This symmetry-breaking step is likely to be a prerequisite for the asymmetrical distribution or activation of ion-transport mechanisms in the FE observed later in development [4].

Participation of bioelectrical signals during axis formation has been demonstrated, e. g., for left-right patterning in Xenopus and chick embryos [22, 23], for lateral embryonic eye patterning in Xenopus [24], and for a-p patterning in planaria $[25,26]$. In particular, the cytoskeleton is an attractive candidate for bioelectrical signalling, since binding of actin-associated factors [27, 28] as well as contractility of actomyosin complexes are controlled by $\mathrm{pH}_{\mathrm{i}}$ [29]. On the other hand, MT are known to amplify electrical signals [30, 31], and modifications of the cytoskeletal organisation have been shown to be promoted by changes in $\mathrm{V}_{\text {mem }}[8,32,33]$.

For some Drosophila mutants with altered axial polarity, connections between morphological polarity and bioelectrical signals have already been described: For example, in egalitarian or Bicaudal-D mutant follicles, where no Ooc and no a-p or d-v polarity is established, aberrant patterns of extracellular ionic currents correlate with disturbed axial polarity [34-36]. In addition, in follicles of the mutant dicephalic, where NC appear at both ends of the Ooc, altered current patterns correlate with impaired a-p polarity [34, 35].

Previous studies on cytoskeletal functions in the FE of Drosophila have revealed the requirement of MT in posterior migration of $\mathrm{BC}$ (to the $\mathrm{Ooc}$ ) and in centripetal migration of FC (between $\mathrm{NC}$ and Ooc) [37]. On the other hand, the organisation of microfilaments (MF) in the FE corresponds to FC differentiation and plays a decisive role in shaping the follicle along its longitudinal axis $[38,39]$. It has also been shown that $\mathrm{pH}_{\mathrm{i}^{-}}$and $\mathrm{V}_{\text {mem }}$-changes induced by several inhibitors of iontransport mechanisms located in the FE [7] simulate naturally occurring bioelectrical changes [4] and lead to alterations of MF- and MT-patterns as observed during FC differentiation [8]. Therefore, gradual modifications of electrochemical signals can serve as physiological means to regulate cell and tissue architecture by modifying cytoskeletal patterns [8].

In the present study, we compare wt and grk follicles with regard to their bioelectrical signals, using a fluorescent $\mathrm{pH}$-indicator and a potentiometric dye $[4,7]$. In addition, we compare wt and grk follicles with regard to their cytoskeletal organisation, using fluorescent phalloidin and an antibody against acetylated $\alpha$-tubulin [8].
Since, in the wt FE, changes in cytoskeletal patterns are linked to changes in bioelectrical properties, it is tempting to analyse correlations between bioelectrical polarity, cytoskeletal polarity and axial polarity in the polarity mutant grk.

\section{Results}

\section{Bioelectrical differences between wt and grk}

Almost all follicles produced by transheterozygous $g r k$ females show morphological defects concerning both axes (Fig. 1). These grk follicles are characterised by a transversally uniform, cuboidal FE covering the Ooc, and by an ON located, predominantly, at the posterior end. This contrasts with wt follicles, where the dorsal side in S10B is defined by a thicker, columnar FE and an anterodorsal position of the ON [11]. These morphological peculiarities correlate with stage-specific differences between wt and grk concerning $\mathrm{V}_{\mathrm{mem}^{-}}$and $\mathrm{pH}_{\mathrm{i}^{-}}$ patterns in the $\mathrm{FE}$, as revealed by the potentiometric dye DiBAC and the pH-indicator CFDA, respectively (Fig. 2). As described earlier [4, 7], stronger fluorescence intensities refer to more depolarised $V_{\text {mem }}$ or more alkaline $\mathrm{pH}_{\mathrm{i}}$, whereas weaker fluorescence intensities refer to more hyperpolarised $\mathrm{V}_{\text {mem }}$ or more acidic $\mathrm{pH}_{\mathrm{i}}$.

During early to mid-vitellogenic stages S8-10A, overall $\mathrm{V}_{\mathrm{mem}^{-}}$and $\mathrm{pH}_{\mathrm{i}}$-patterns of wt (Fig. 2a-c and $\mathrm{m}-\mathrm{o}$ ) and grk (Fig. 2g-i and s-u) are rather similar, since d-v gradients have not yet emerged in the wt (cf. [4, 7]). In both wt and $g r k$, the somatic FE in S8 is more depolarised and more acidic than the germline cells (Ooc and NC). During S9$10 \mathrm{~A}$, grk follicles develop a similar a-p $\mathrm{V}_{\text {mem-pattern as }} \mathrm{wt}$ follicles, the mainbody follicle cells $(\mathrm{mFC})$ being hyperpolarised in relation to the neighbouring $\mathrm{pFC}$ and centripetal follicle cells (cFC). In addition, during S9-10A, a-p $\mathrm{pH}_{\mathrm{i}}-$ gradients are present in the FE of both wt and grk, the pFC being most alkaline. Additionally, in both genotypes, the anterior-most $\mathrm{NC}$ is the most alkaline.

However, in the S9 FE of wt and grk, a closer look reveals that the slopes of the a-p $V_{\text {mem-gradients differ }}$ (Fig. 2b and $\mathrm{h}$; for variability between follicles of the same stage, see Additional file: Fig. S1). Compared to wt, the $\mathrm{mFC}$ in $g r k$ are less hyperpolarised in relation to neighbouring $\mathrm{cFC}$, and the whole $\mathrm{FE}$ is more depolarised, resulting in a shallower a-p $\mathrm{V}_{\text {mem }}$-gradient (significantly reduced angle of gradient; Table 1 and Fig. 3a).

Even more striking differences become obvious during S10B, when d-v electrochemical gradients are established in the FE of wt (Fig. 2d and p) but not grk (Fig. $2 j_{1}$ and v; Table 2 and Fig. 3b; for variability between follicles of the same stage, see Additional file: Fig. S1). In most analysed wt S10B follicles, according to the position of the $\mathrm{ON}$, the more depolarised or more alkaline side was identified as the ventral side (cf. [4, 7]). In some grk $\mathrm{S} 10 \mathrm{~B}$ follicles, a transversal $\mathrm{V}_{\text {mem }}$-gradient was detected 


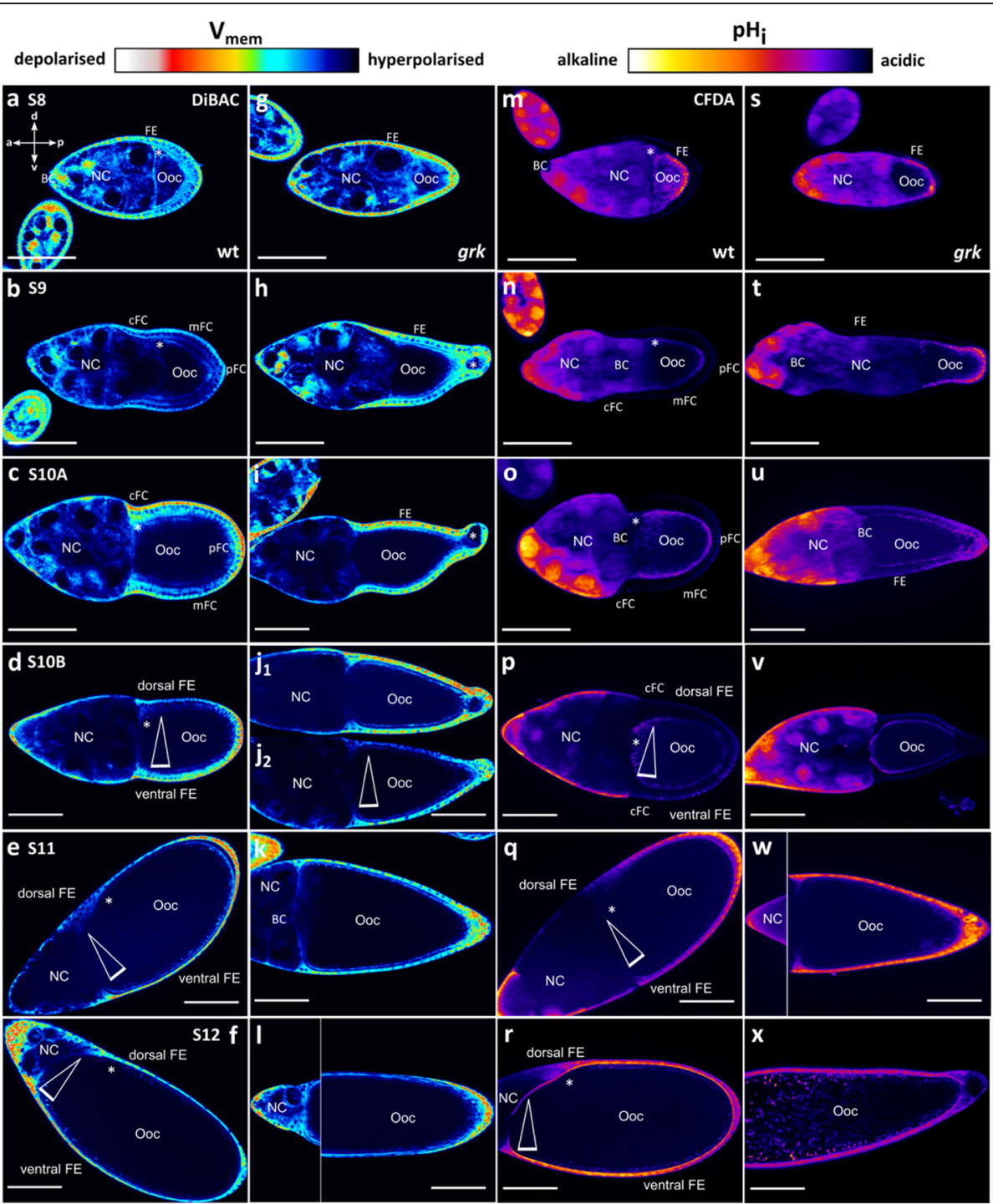

Fig. 2 Typical dorsoventral electrochemical gradients, as observed in the wt FE beginning with S10B, are missing in grk. DiBAC staining ( $V_{\text {mem, }}$ a-I) and CFDA staining $\left(\mathrm{pH}_{\mathrm{i}}, \mathbf{m}-\mathbf{x}\right)$; median optical sections (SIM) of typical S8-12 follicles. a-I Pseudocolor images of DiBAC stained wt (a-f) and grk $(\mathbf{g}-\mathbf{I})$ follicles. Relative depolarisation of $\mathrm{V}_{\text {mem }}$ is indicated by stronger (red), relative hyperpolarisation by weaker (blue) fluorescence intensities (scale bars represent $100 \mu \mathrm{m}$; composed pictures show different regions of the same follicle; positions of the ON are marked with asterisks). $\mathbf{m}$ - $\mathbf{x}$ Pseudocolor images of CFDA stained wt $(\mathbf{m}-\mathbf{r})$ and $g r k(\mathbf{s}-\mathbf{x})$ follicles. Relatively alkaline $\mathrm{pH}_{\mathrm{i}}$ is indicated by stronger (yellow), relatively acidic $\mathrm{pH}$ weaker (blue) fluorescence intensities. In early to mid-vitellogenic S8-10A (a-c, $\mathbf{g}-\mathbf{i}, \mathbf{m}-\mathbf{o}, \mathbf{s}-\mathbf{u}$ ), a-p gradients, but no d- $\mathbf{v}$ gradients, of both $V_{\text {mem }}$ and $\mathrm{pH}_{\mathrm{i}}$ are detectable in the $\mathrm{FE}$ of both wt and grk. Compared to $\mathrm{wt}$, the $\mathrm{mFC}$ in grk are less hyperpolarised relative to $\mathrm{CFC}$, and the whole $\mathrm{FE}$ is more depolarised. More striking differences between wt and grk become obvious during S1OB when $\mathrm{d}-\mathrm{v}$ gradients (triangles indicate fluorescence-intensity gradients) of both $\mathrm{V}_{\text {mem }}$ and $\mathrm{pH}_{\mathbf{i}}$ are established in wt $(\mathbf{d}, \mathbf{p})$, but not in grk $\left(\mathbf{j}_{1}, \mathbf{v}\right)$. Some grk S10B follicles showed a transversal $V_{\text {mem-gradient }}\left(\mathbf{j}_{2}\right)$, but such a gradient was never observed during later stages $(\mathbf{k}, \mathbf{l})$, in contrast to wt $(\mathbf{e}, \mathbf{f})$. For variability of follicles in S9 and S10B, see Additional file: Fig. S1; for numbers of analysed follicles, see Additional file: Table S3

(Fig. $2 \mathrm{j}_{2}$; Additional file: Table S1) but, in contrast to wt, such a gradient was absent from later stages (S11-12; Fig. 2e,f and $\mathrm{k}, \mathrm{l})$. A transversal $\mathrm{pH}_{\mathrm{i}}$-gradient, however, was never observed in grk (Fig. 2v-x; Table 2 and Fig. 3b; Additional file: Table S1). Concerning a-p electrochemical gradients, significant differences between wt and grk were not observed (Table 2 and Fig. 3c; Additional file: Table S2).

\section{Cytoskeletal differences between wt and grk}

Using fluorescent phalloidin and an antibody against acetylated $\alpha$-tubulin, we compared, during S8-12, the FE of wt and grk concerning cytoskeletal organisation (Figs. 4 and 5; for wt, cf. [8]). During S8, basal microfilaments (bMF) show the same parallel transversal alignment in wt and grk (Fig. 4a,g). Except for wt S10A (Fig. 4c), this alignment is missing in wt S9, S10B and S11, 
Table 1 In the S9 FE of $g r k$, the a-p $V_{\text {mem }}$-gradient is shallower

\begin{tabular}{lll}
\hline & $\mathbf{V}_{\text {mem }}$ & $\mathrm{pH}_{\mathbf{i}}$ \\
\hline Gradient & Fraction of S9 follicles & Fraction of S9 follicles \\
a-p & with $\mathrm{cFC} / \mathrm{mFC} \geq \mathbf{1 . 5 ^ { \S }}$ & with $\mathrm{pFE} / \mathrm{aFE} \geq \mathbf{1 . 3 ^ { \# }}$ \\
wt & $5 / 5$ & $2 / 5$ \\
grk & $0 / 5$ & $2 / 5$ \\
\hline
\end{tabular}

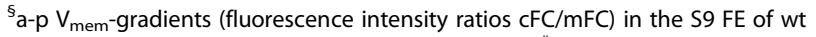
and grk were evaluated as described previously [7]. ${ }^{*}$ a-p $\mathrm{pH}_{\mathrm{i}}$-gradients

(fluorescence intensity ratios pFE/aFE) were quantified according to Fig. $1 e_{2}$. The higher the fluorescence intensity ratio is, the steeper is the gradient $(n=5$; see also Fig. 3a)

but it persists during these stages in grk. In all cFC in wt $\mathrm{S} 9$, and in dorsal cFC in wt S10B, condensations of bMF appear (Fig. 5; cf. [8]). This phenomenon is accompagnied by a loss of the transcellular parallel alignment of bMF in the remaining wt FE (Fig. 4b, d; for variability between follicles of the same stage, see Additional file: Fig. S2). In wt S11, a rearrangement of bMF occurs with fan-shaped structures (Fig. 4e; cf. [8]), and in wt S12, a dense transversal pattern of parallel bMF develops (Fig. 4f). In grk S11, many FC seem to contain no bMF (Fig. 4k). Moreover, in several grk S12 follicles, bMF were almost totally missing, while other follicles exhibit transversally aligned bMF (Fig. 4l). Thus, as observed for bioelectrical properties, the grk FE exhibits striking stage-specific pecularities concerning the bMF-pattern. In contrast to wt, grk bMF are characterised by transversal alignment during all analysed stages, while wt-typical condensations in cFC (S9) and dorsal cFC (S10B) are absent (Fig. 4g-l; Fig. 5).

During S8, all wt FC show a more or less cellautonomous organisation of MT, being arranged around the nuclei (Fig. $4 \mathrm{~m}$ ). Beginning with $S 9$, the MT in wt cFC and mFC develop a longitudinal alignment, while the MT in pFC maintain their cell-autonomous arrangement (Fig. 4n). The longitudinal MT-alignment begins in $\mathrm{cFC}$ and spreads out over mFC to pFC (cf. [8]). However, in grk, in the whole FE, longitudinal alignment of MT was observed during all analysed stages (Fig. 4s-x). Between neighbouring FC, this MT-alignment in grk appears to be more coordinated than in wt. Thus, as observed for bMF, the wt MT-pattern (Fig. 4m-r) is more cell-autonomously organised, whereas the grk MTpattern is characterised by a coordinated transcellular organisation along the longitudinal axis (Fig. 4s-x).

The characteristic bioelectrical and cytoskeletal features of wt and grk in S9 and S10B are summarised in Fig. 6. In early vitellogenic stages (up to S9), a-p gradients of $\mathrm{V}_{\text {mem }}$ and $\mathrm{pH}_{\mathrm{i}}$ show the same polarity in both genotypes. However, the a-p $\mathrm{V}_{\text {mem-gradient in }}$ grk $\mathrm{S} 9$ is shallower, and the whole FE is more depolarised compared to wt. In grk S9, bMF are characterised by transversal alignment and MT by longitudinal alignment, whereas both wt-typical condensations of bMF and cellautonomously organised MT are absent. During S10B, striking bioelectrical as well as cytoskeletal differences appear, when $\mathrm{d}-\mathrm{v}$ polarity becomes obvious in wt but not in grk. In wt S10B, prominent a-p and d-v gradients of both $\mathrm{V}_{\text {mem }}$ and $\mathrm{pH}_{\mathrm{i}}$ appear in combination with condensations of bMF in dorsal cFC and cell-autonomously organised MT in $\mathrm{mFC}$ and pFC. In grk S10B, however, $\mathrm{d}-\mathrm{v} \mathrm{V}_{\text {mem }^{-}}$and $\mathrm{pH}_{\mathrm{i}}$-gradients are missing and both condensations of bMF and cell-autonomously organised MT are absent (Fig. 6).

\section{Discussion}

\section{Altered axial polarity correlates with altered electrochemical gradients}

At the posterior pole of $g r k$ follicles older than S9, migrating $\mathrm{FC}$ can be observed which, more or less, enclose the ON. According to previous reports $[9,17]$, the three anterior FC types (BC, stretched FC and $\mathrm{CFC}$ ) are duplicated at the posterior end of $g r k$ follicles. The "posterior FC" in grk undergo similar morphological movements as the anterior FC. "Posterior $\mathrm{BC}$ " lose their epithelial organisation, "adjacent posterior FC" become stretched and "posterior cFC" migrate centripetally, sometimes even bisecting the Ooc. These aberrations of axial polarity in the FE of grk correlate with altered bioelectrical and cytoskeletal patterns as described in the present study.

In the FE of wt and grk, we compared stage-specific longitudinal and transversal gradients of $\mathrm{V}_{\text {mem }}$ and $\mathrm{pH}_{\mathrm{i}}$, respectively. Since d-v electrochemical gradients are not yet established in the wt during early to mid-vitellogenic stages S8-10A (cf. [4, 7]), the overall $\mathrm{V}_{\mathrm{mem}^{-}}$and $\mathrm{pH}_{\mathrm{i}}$-patterns of wt and grk are rather similar. However, corresponding to impaired a-p polarity in $g r k$, the slope of the a-p $V_{\text {mem-gradient }}$ in $S 9$ is significantly reduced compared to wt.

More striking bioelectrical characteristics relating to missing d-v polarity in grk appear in S10B. During grk S10B-12, significant transversal electrochemical gradients are absent. In early wt S10B, the FE becomes continuously depolarised from dorsal to ventral while $\mathrm{pH}_{\mathrm{i}}$ increases in the same direction. During late wt S10B-12, dorsal cFC show increasing depolarisation (cf. [7]). Refering to morphological variability, some grk S10B follicles exhibit a transient transversal $V_{\text {mem-gradient which }}$ was never observed during later stages.

\section{Altered electrochemical gradients correlate with altered cytoskeletal patterns}

As shown in detail recently [8], stage-specific alterations of $\mathrm{V}_{\text {mem }}$ and $\mathrm{pH}_{\mathrm{i}}$ correlate with structural modifications of bMF and MT in the wt FE. Higher $\mathrm{pH}_{\mathrm{i}}$, as observed in $\mathrm{mFC}$ and $\mathrm{pFC}$ in $\mathrm{S} 10 \mathrm{~B}$, stabilises the parallel 
a $\quad$ S9

$\mathrm{n}=\mathbf{5}$ Anteroposterior $\mathrm{V}_{\text {mem }}$ gradients

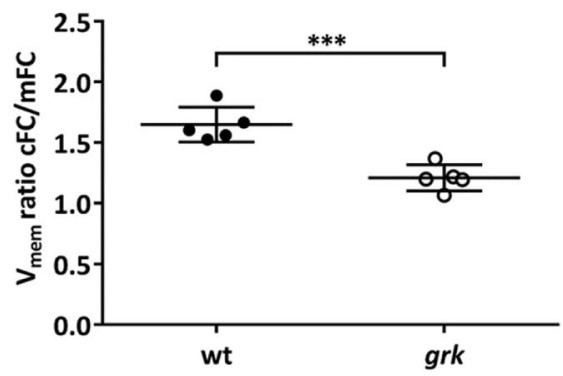

b S10B

$\mathrm{n}=7 \quad$ Transversal $\mathrm{V}_{\text {mem }}$ gradients

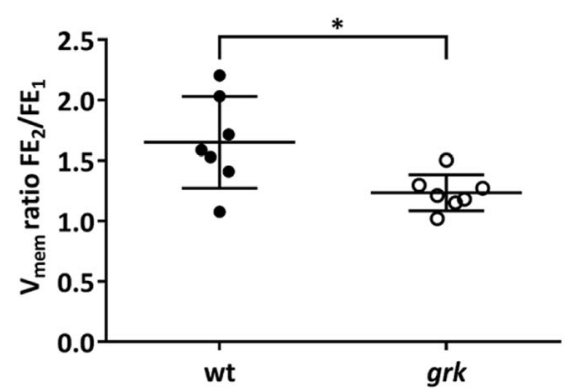

C $\mathrm{S10B}$

$n=7 \quad$ Anteroposterior $V_{\text {mem }}$ gradients

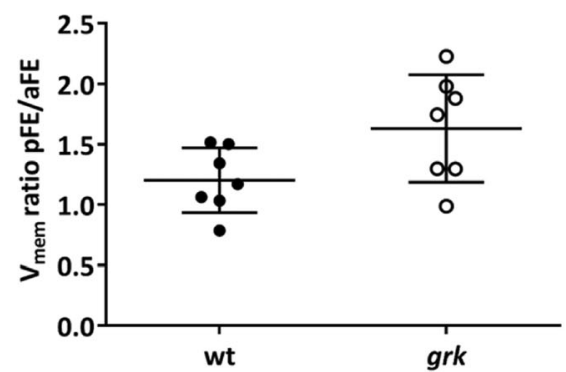

Anteroposterior $\mathrm{pH}_{\mathrm{i}}$ gradients

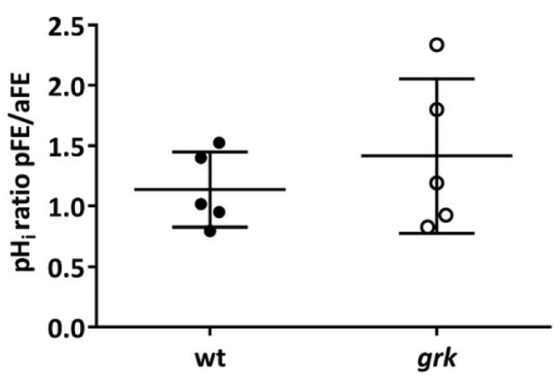

Transversal $\mathrm{pH}_{\mathrm{i}}$ gradients

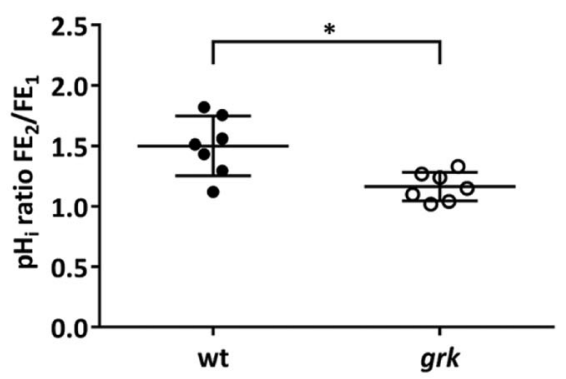

Anteroposterior $\mathrm{pH}_{\mathrm{i}}$ gradients

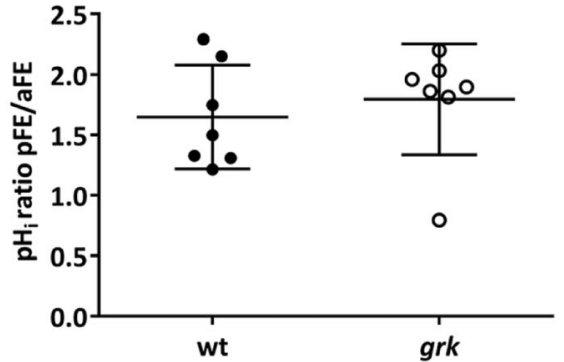

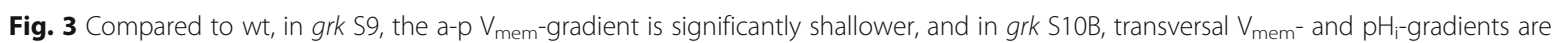

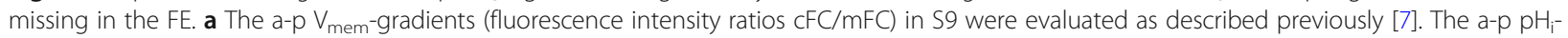
gradients (fluorescence intensity ratios pFE/aFE) were quantified according to Fig. 1 $e_{2}$. The higher the fluorescence intensity ratio is, the steeper is the gradient. Mean values ( $\pm \mathrm{SD}$, standard deviation) were compared using an unpaired t-test. Difference for $\mathrm{V}_{\text {mem-gradients }}$ is significant (*** $p<$

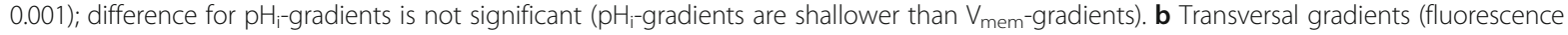
intensity ratios $\mathrm{FE}_{2} / \mathrm{FE}_{1}$; larger value vs. smaller value), and $\mathbf{c}$ a-p gradients (fluorescence intensity ratios $\mathrm{pFE} / \mathrm{aFE}$ ) in S1OB were quantified as shown in Fig. 1e. Differences for transversal gradients are significant ( $\left.{ }^{*} p<0.05\right)$; differences for a-p gradients are not significant

alignment of bMF and results in loss of the longitudinal alignment of MT (leading to a more cell-autonomous MT-arrangement). Lower $\mathrm{pH}_{\mathrm{i}}$, as observed in dorsal cFC in early S10B, leads to increasing disorder and condensation of bMF as well as to stabilisation of the longitudinal MT-alignment. Lower $\mathrm{pH}_{\mathrm{i}}$ in combination with relatively depolarised $\mathrm{V}_{\text {mem }}$, as observed in dorsal $\mathrm{cFC}$ in late S10B, contributes to the disintegration of bMF. Correlations between bioelectrical properties and cytoskeletal patterns, as observed in different stages and different regions of the wt FE, correspond to correlations induced by inhibitors of various ion-transport mechanisms [8]. These observations lend support to the hypothesis that gradual modifications of electrochemical signals can serve as physiological means to regulate cell and tissue architecture by modifying cytoskeletal patterns $[7,8]$.

Further support to this hypothesis is provided by the present study. Shallower (or no) $\mathrm{V}_{\text {mem-gradients and }}$ 
Table 2 In the S1OB FE of grk, distinct transversal $\mathrm{V}_{\mathrm{mem}}$ - and $\mathrm{pH}_{\mathrm{i}}$-gradients are missing

\begin{tabular}{|c|c|c|}
\hline & $V_{\text {mem }}$ & $\mathrm{pH}_{\mathrm{i}}$ \\
\hline $\begin{array}{l}\text { Gradients } \\
\text { transversal }^{\S}\end{array}$ & $\begin{array}{l}\text { Fraction of } \mathrm{S} 10 \mathrm{~B} \text { follicles } \\
\text { with } \mathrm{FE}_{2} / \mathrm{FE}_{1} \geq 1.5\end{array}$ & $\begin{array}{l}\text { Fraction of } \mathrm{S} 10 \mathrm{~B} \text { follicles } \\
\text { with } \mathrm{FE}_{2} / \mathrm{FE}_{1} \geq 1.5\end{array}$ \\
\hline wt & $5 / 7$ & $4 / 7$ \\
\hline grk & $1 / 7$ & $0 / 7$ \\
\hline$a-p^{\#}$ & with $\mathrm{pFE} / \mathrm{aFE} \geq 1.5$ & with $\mathrm{pFE} / \mathrm{aFE} \geq 1.5$ \\
\hline wt & $2 / 7$ & $4 / 7$ \\
\hline grk & $4 / 7$ & $6 / 7$ \\
\hline
\end{tabular}

${ }^{5}$ Transversal gradients (fluorescence intensity ratios $\mathrm{FE}_{2} / \mathrm{FE}_{1}$; larger value vs. smaller value), and " $\mathrm{a}$ - $\mathrm{p}$ gradients (fluorescence intensity ratios $\mathrm{pFE} / \mathrm{aFE}$ ) in the S10B FE of wt and grk were quantified as shown in Fig. 1e (for variability see Additional file: Tables S1 and S2). The higher the fluorescence intensity ratio is, the steeper is the gradient ( $n=7$; see als Fig. $3 \mathrm{~b}$ and c). Since, in grk, the cFC and $\mathrm{mFC}$ (refering to aFE) are often both more hyperpolarised and more acidic than the aberrant pFC, a-p gradients in $g r k$ seem to be somewhat steeper than in wt (cf. Additional file: Fig. S1 and Table S2), but this difference was not significant (cf. Fig. 3c)

relative alkalisation, as generated by the inhibition of certain ion-transport mechanisms [7], lead to stabilisation of the parallel transversal bMF-pattern in wt [8]. This also holds true for the cFC in grk S9 and the dorsal cFC in grk S10B, where bMF retain their transversal alignment while wt-typical condensation and subsequent disintegration of bMF are missing. Similarly, shallower (or no) $\mathrm{V}_{\mathrm{mem}}$-gradients lead to stabilisation of the longitudinal MT-orientation in wt [8]. This also holds true for $\mathrm{mFC}$ and pFC in grk S9 as well as in $g r k \mathrm{S10B}$, where, in addition, a transversal $\mathrm{pH}_{\mathrm{i}}$-gradient is missing. In the whole grk FE, MT exhibit a longitudinal transcellular alignment, whereas in wt $\mathrm{mFC}$ and pFC, MT-patterns are characterised by a more cell-autonomous organisation.

In $g r k$, preferential alignment of bMF along the transversal axis and of MT along the longitudinal axis is obviously enhanced. Assuming a duplication of anterior FC types at the "posterior pole" in $\operatorname{grk}[9,14,17]$, it seems plausible that both the transversal alignment of bMF and the longitudinal alignment of MT, as found in the anterior FE of $g r k$, is duplicated in the "posterior FE".

\section{Bioelectrical and cytoskeletal polarity depend on axial polarity}

Considering the described wt- and grk-specific bioelectrical and cytoskeletal features (for summary, see Fig. 6), it is obvious that bioelectrical and cytoskeletal polarity are linked to axial polarity. The establishment of electrochemical gradients in the FE depends on asymmetrically distributed or activated ion-transport mechanisms and gap junctions $[4,7,40-44]$. This asymmetry is presumed to depend on early Grk-Egfr signalling and continues to exert influence on cytoskeletal patterns later in development $[7,8]$.
In wt S9, longitudinal electrochemical gradients with relative depolarisation and relative acidification in $\mathrm{cFC}$ result in condensation of bMF and in longitudinally aligned MT in this area [8]. Impaired a-p polarity in grk S9, however, leads to relative depolarisation in the whole FE resulting in a shallower longitudinal $\mathrm{V}_{\text {mem }}$-gradient and in stabilisation of the transversal bMF-pattern. In wt S10B, strong transversal electrochemical gradients, showing relative hyperpolarisation and relative acidification in dorsal cFC, lead to condensation and disintegration of bMF [8]. On the other hand, as a consequence of missing $\mathrm{d}-\mathrm{v}$ polarity in grk $\mathrm{S} 10 \mathrm{~B}$, transversal electrochemical gradients as well as bMF-condensation and disintegration are absent from the whole FE.

Therefore, we propose that shallow or missing electrochemical gradients, as observed in grk, result in stabilisation of cytoskeletal patterns. Throughout oogenesis, the bMF remain oriented along the transversal axis while the MT remain oriented along the longitudinal axis. This interpretation corresponds to the previous observation that experimentally reduced $\mathrm{V}_{\text {mem }}$-gradients stabilise both bMF- and MT-patterns [8].

\section{Conclusion}

Our analysis of the Drosophila mutant grk leads to the conclusion that not only cell-specific levels of $\mathrm{V}_{\text {mem }}$ and $\mathrm{pH}_{\mathrm{i}}$, or the polarities of electrochemical gradients [8], but also the slopes of these gradients are crucial for either alteration or stability of cytoskeletal patterns. When primary signals of axial polarity, like Grk, are weak or missing, ion-transport mechanisms and gap junctions in the FE are not distributed or activated asymmetrically. Consequently, electrochemical gradients are shallow and patterns of cytoskeletal elements remain unchanged.

\section{Methods \\ Fly stocks}

For analysis, Drosophila melanogaster wild-type Oregon $\mathrm{R}$ (wt) and gurken (grk) were used. The strains w; $\operatorname{grk}^{H F 48} / \mathrm{CyO}$ and $w ; \operatorname{grk}^{2 B 6} / \mathrm{CyO}$ (gift of S. Roth and O. Karst, Köln, Germany) were crossed to generate transheterozygous $g r k^{H F 48} / g r k^{2 B 6}$ flies. Although $g_{r k}^{2 B 6}$ is the strongest existing allele $[14,45,46]$, only a combination of both grk null alleles led to a penetrance of $100 \%$ ventralised grk follicles (Fig. 1c). Flies were reared at $25^{\circ} \mathrm{C}$ in the dark on standard food with additional fresh yeast.

\section{Preparation of follicles}

Females were killed by crushing the head with tweezers without anaesthesia, and 3 days old wt or 2 days old grk ovaries were dissected (older grk ovaries contained many degenerating follicles). Single follicles of stages S8-12 were isolated from the epithelial sheath by pulling at the anterior tip of an ovariole. Dissection was carried out in 


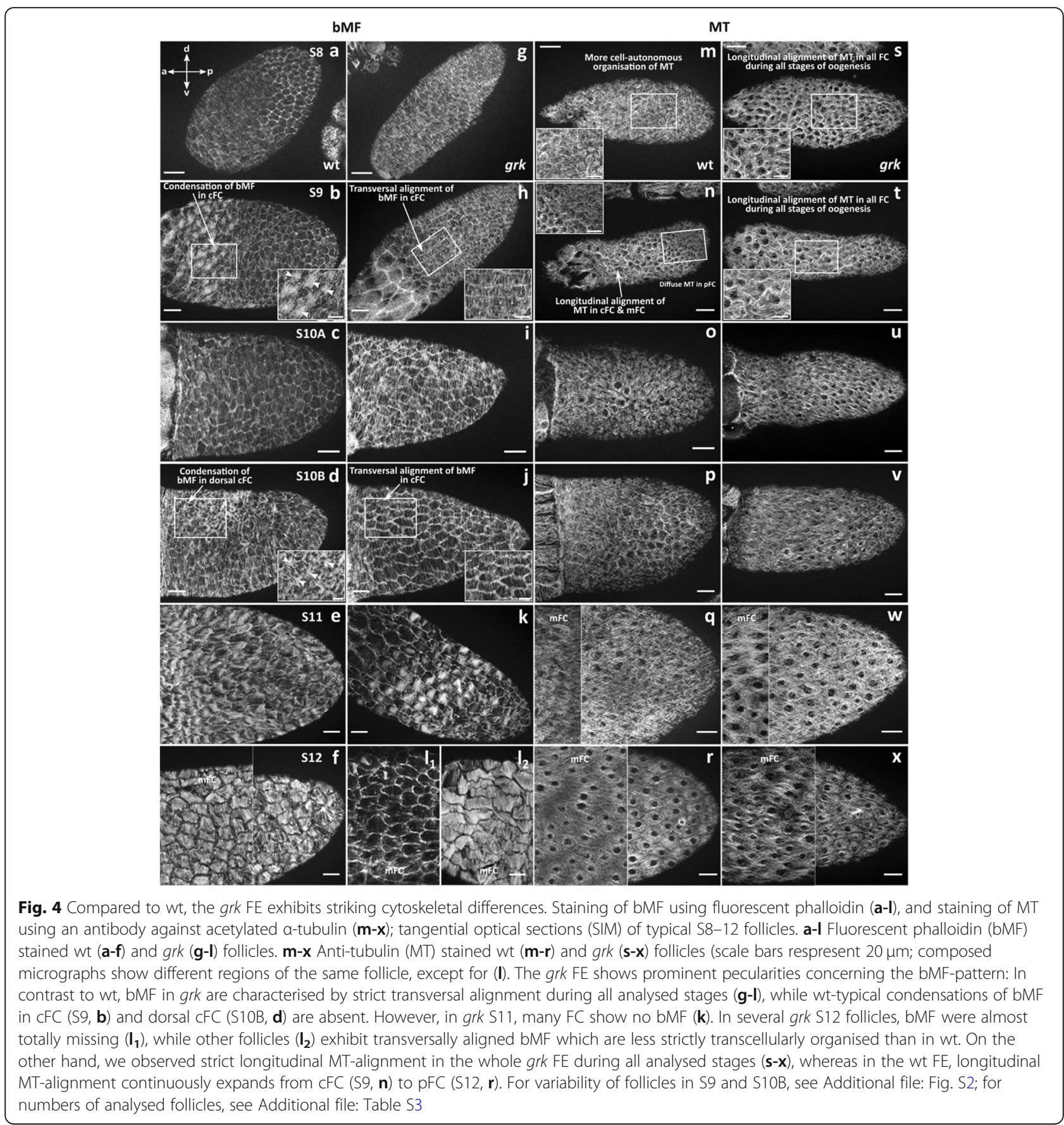

Drosophila phosphate-buffered saline [47]. For staining with fluorescent indicators, we used R-14 Medium [47] which is best suited for in-vitro culture of Drosophila follicles [48].

\section{Fluorescent membrane potential indicator}

For the analysis of $\mathrm{V}_{\text {mem-patterns, we used the fluores- }}$ cent potentiometric dye $\mathrm{DiBAC}\left(\mathrm{DiBAC}_{4}(3)\right.$; bis-(1,3dibutylbarbituric acid) trimethine oxonol; Molecular Probes/Thermo Fisher Scientific, USA). As described earlier [4, 7], relative depolarisation leads to intracellular accumulation of the anionic dye and to increasing fluorescence while relative hyperpolarisation leads to decreasing fluorescence. Living follicles were incubated for 20 min in R-14 medium containing $4 \mu \mathrm{M}$ DiBAC (dissolved in $70 \%$ ethanol). Thereafter, they were mounted in R-14 medium and analysed immediately using $\times 10 / 0.25$ and $\times 20 / 0.5$ objectives and median optical sections (Fig. 1d) on a Zeiss AxioImager.M2 structured-illumination microscope (SIM), equipped with a Zeiss ApoTome, a 
a

S9

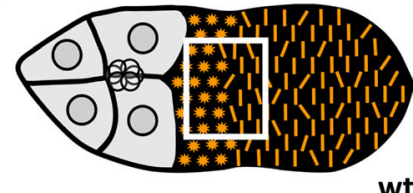

b

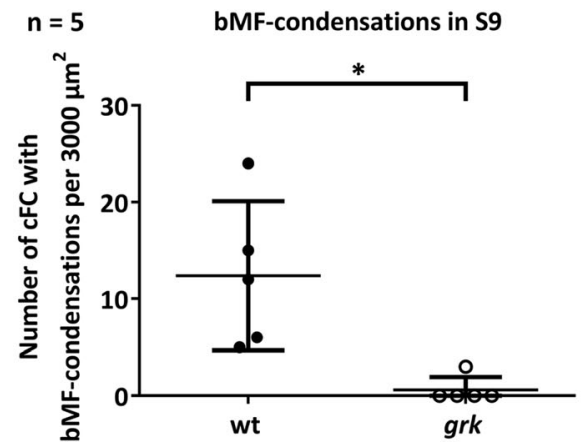

S10B
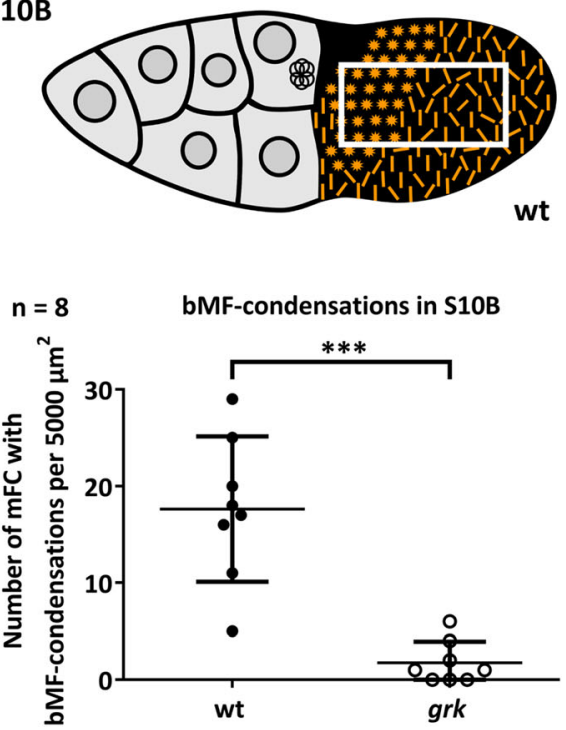

Fig. 5 Comparison of wt and grk concerning condensations of bMF in S9 (cFC) and S10B (mFC). Wt-typical condensations (for regions of interest, see boxes marked in $\mathbf{a})$ are missing in $\operatorname{grk}(\mathbf{b})$. Mean values ( $\pm \mathrm{SD}$, standard deviation) were compared using an unpaired t-test $\left(^{*} p<0.05\right.$; $\left.{ }^{* *} p<0.001\right)$

Zeiss AxioCamMRm camera and the appropriate filter set. For numbers of analysed S8-12 follicles, see Additional file: Table S3.

\section{Fluorescent intracellular $\mathrm{pH}$ indicator}

For the analysis of $\mathrm{pH}_{\mathrm{i}}$-patterns, we used the fluorescent pH-indicator CFDA (5-CFDA,AM; 5-carboxyfluorescein diacetate, acetoxymethyl ester; Molecular Probes) which enters cells as an anion. As described earlier [4, 7], increasing fluorescence indicates relative alkalisation while decreasing fluorescence due to protonation indicates relative acidification. Living follicles were incubated for 20 min in R-14 medium containing $4 \mu \mathrm{M}$ CFDA (dissolved in dimethyl sulfoxide, DMSO). Subsequently, the follicles were mounted in R-14 medium and viewed immediately as described above using median optical sections (Fig. 1d). For numbers of analysed S8-12 follicles, see Additional file: Table S3.

\section{F-actin staining using fluorescent phalloidin}

Follicles were fixed in microfilament-stabilising buffer (MF-buffer $[8,38]$ ) with $4 \%$ formaldehyde and $0.2 \%$ Triton X-100 for $20 \mathrm{~min}$ at room temperature, washed with phosphate-buffered saline (PBS) and stained with $0.25 \mu \mathrm{g} / \mathrm{ml}$ phalloidin-FluoProbes $550 \mathrm{~A}$ (Interchim, France; dissolved in DMSO) in PBS. After washing, the follicles were mounted in Fluoromount $G$ (Interchim) and viewed as described above using a $\times 40 / 1.3$ oil objective and tangential optical sections (Fig. 1d). For numbers of analysed S8-12 follicles, see Additional file: Table S3.
Indirect immunofluorescence staining of microtubules Follicles were fixed for $20 \mathrm{~min}$ at room temperature in MF-buffer as described above, washed with PBS containing $0.1 \%$ Triton X-100 and blocked for $1 \mathrm{~h}$ at room temperature with $2 \%$ bovine serum albumin (BSA) $/ 0.1 \%$ Triton X-100 in PBS. Thereafter, the follicles were incubated overnight at $4{ }^{\circ} \mathrm{C}$ or for $1 \mathrm{~h}$ at room temperature in PBS containing 1\% BSA $/ 0.1 \%$ Triton X-100 and a monoclonal antibody against acetylated $\alpha$-tubulin (6-11B-1; Santa Cruz Biotechnology, USA) diluted 1:100 [8]. After washing, the follicles were treated for $1 \mathrm{~h}$ at room temperature with goat anti-mouse-biotin (Dianova, Germany) diluted 1:200 in PBS containing $1 \%$ BSA $/ 0.1 \%$ Triton X-100. Washing was repeated before the follicles were incubated for $30 \mathrm{~min}$ with streptavidin-TexasRed (Dianova) diluted 1:100 in PBS containing 1\% BSA/0.1\% Triton X100. After washing, the follicles were mounted and analysed as described above using tangential optical sections (Fig. 1d). For numbers of analysed S8-12 follicles, see Additional file: Table S3. Controls were performed without primary antibody.

\section{Staging of follicles and determination of axes}

Follicles were staged according to criteria described previously $[11,49]$. To determine the a-p axis, the anterior position of the NC was used as marker, while for the d-v axis, the anterodorsal position of the $\mathrm{ON}$ and the columnar dorsal FE (S10B) were used. For grk follicles, the same criteria for staging and axis determination were applied. Due to the posterior location of the $\mathrm{ON}$ and the 


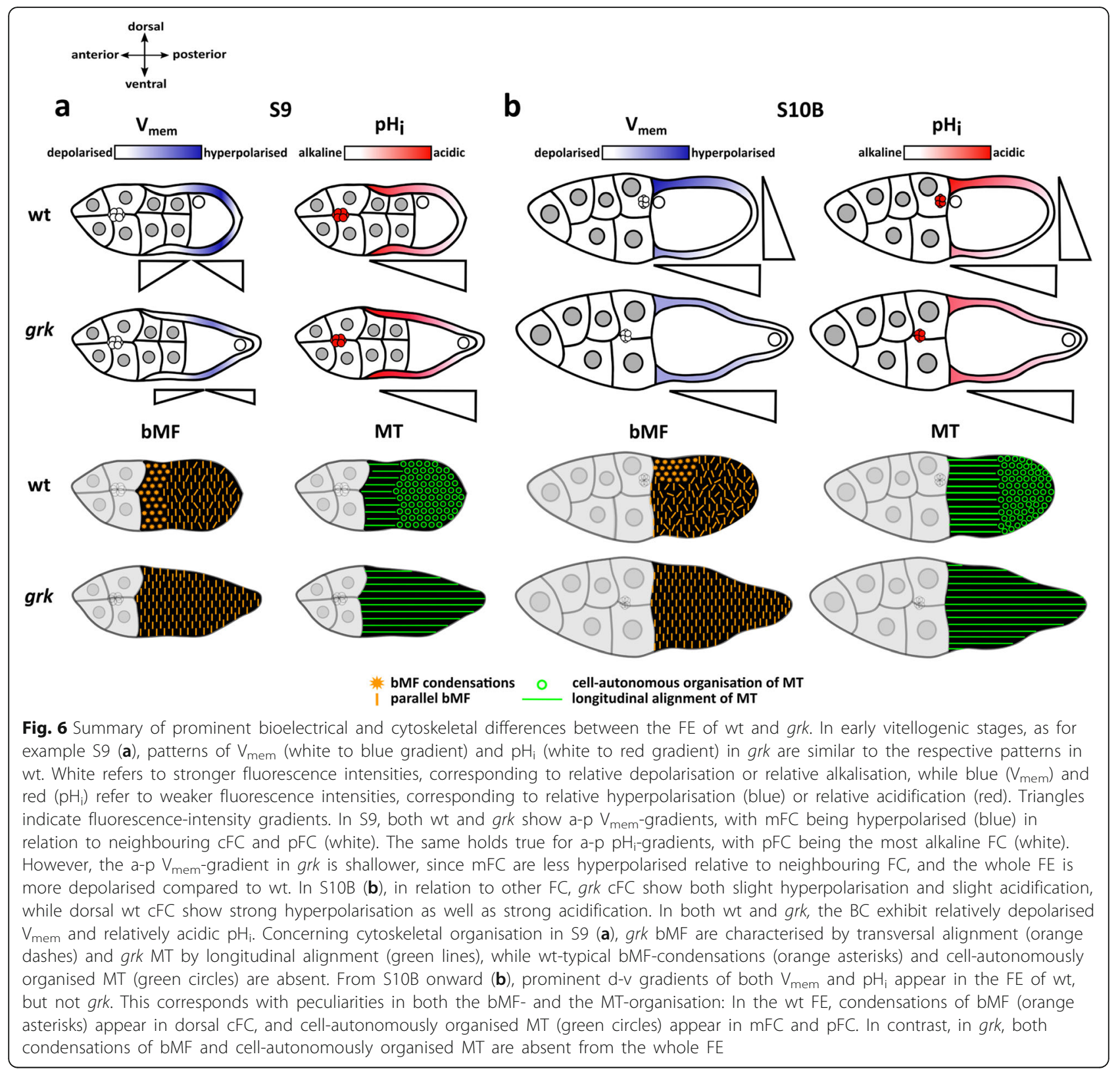

transversally homogeneous FE, no dorsal side was detectable in grk (Fig. 1).

\section{Quantification of fluorescence intensities in the FE}

To quantify both the longitudinal and the transversal $\mathrm{V}_{\text {mem }}{ }^{-}$and $\mathrm{pH}_{\mathrm{i}}$-gradients in the FE of grk and wt, respectively, we used median optical sections (Fig. 1d) of stained follicles. Fluorescence itensities ("mean grey value") of both sides $\left(\mathrm{FE}_{1}\right.$ and $\mathrm{FE}_{2}$ or aFE and pFE) were measured using ImageJ (Fig. 1e) and a ratio of both values was determined. For a-p $V_{\text {mem }^{-}}$ gradients in S9, fluorescence intensities of $\mathrm{cFC}, \mathrm{mFC}$ and $\mathrm{pFC}$ were measured separately and ratios determined according to [7].

\section{Supplementary information}

Supplementary information accompanies this paper at https://doi.org/10. 1186/s12861-020-00210-8.

Additional file 1: Figure S1. Typical dorsoventral electrochemical gradients, as observed in the wt FE beginning with S10B, are absent in grk. Additional examples corresponding to Fig. 2, showing the variability between follicles of the same stage. Table S1. Quantification of fluorescence intensities of transversal electrochemical gradients in the FE of wt and grk (S10B). Data corresponding to Table 2 and Fig. 3b. Table S2. Quantification of fluorescence intensities of anteroposterior electrochemical gradients in the FE of wt and grk (S10B). Data corresponding to Table 2 and Fig. 3c. Figure S2. The grk FE exhibits striking cytoskeletal differences compared to wt (S9 and S10B). Additional examples corresponding to Fig. 4, showing the variability between follicles of the same stage. Table S3. Numbers of follicles analysed for each condition and developmental stage. 


\section{Abbreviations}

5-CFDA,AM: 5-Carboxyfluorescein diacetate, acetoxymethyl ester; aFE: Anterior half of the FE; a-p: Anteroposterior; BC: Border cells; bMF: Basal microfilaments; BSA: Bovine serum albumine; CFC: Centripetal follicle cells; $\mathrm{DiBAC}_{4}(3)$ : Bis-(1,3-dibutylbarbituric acid) trimethine oxonol; DMSO: Dimethyl sulfoxide; d-v: Dorsoventral; FC: Follicle cells; FE: Follicular epithelium; grk: gurken; MF: Microfilaments; mFC: Mainbody follicle cells; MT: Microtubules; NC: Nurse cells; ON: Oocyte nucleus; Ooc: Oocyte; PBS: Phosphate-buffered saline; pFC: Posterior follicle cells; pFE: Posterior half of the $\mathrm{FE}_{;} \mathrm{pH}_{\mathrm{i}}$ : Intracellular $\mathrm{pH}$; S: Stage; SIM: Structured-illumination microscopy; $V_{\text {mem }}$ : Membrane potential; wt: Wild-type

\section{Acknowledgements}

We are indepted to Siegfried Roth and Oliver Karst (Köln, Germany) for providing the grk strains, and to Isabel Weiß for technical assistance.

\section{Authors' contributions}

SS carried out the experiments and analysed the data under the supervision of JB. JB conceived the study and reviewed the data. Both authors wrote the manuscript and read and approved the final version.

\section{Funding}

Financial support by RWTH Aachen University is acknowledged. The funding body played no role in the design of the study or the collection, analysis, and interpretation of data, or in writing the manuscript.

\section{Availability of data and materials}

The datasets used during the current study are available from the corresponding author on reasonable request.

\section{Ethics approval and consent to participate} Not applicable.

\section{Consent for publication}

Not applicable.

\section{Competing interests}

The authors declare that they have no competing interests.

\section{Received: 19 November 2019 Accepted: 26 February 2020}

\subsection{0.}

\section{References}

1. Campetelli A, Bonazzi D, Minc N. Electrochemical regulation of cell polarity and the cytoskeleton. Cytoskeleton. 2012;69:601-12.

2. Adams DS, Levin M. Endogenous voltage gradients as mediators of cell-cell communication: strategies for investigating bioelectrical signals during pattern formation. Cell Tissue Res. 2013;352:95-122.

3. Chang F, Minc N. Electrochemical control of cell and tissue polarity. Annu Rev Cell Dev Biol. 2014;30:317-36.

4. Krüger J, Bohrmann J. Bioelectric patterning during oogenesis: stage-specific distribution of membrane potentials, intracellular $\mathrm{pH}$ and ion-transport mechanisms in Drosophila ovarian follicles. BMC Dev Biol. 2015;15:1.

5. Cervera J, Manzanares JA, Mafe S. Cell-cell bioelectrical interactions and local heterogeneities in genetic networks: a model for the stabilization of single-cell states and multicellular oscillations. Phys Chem Chem Phys. 2018; 20:9343-54.

6. Pietak A, Levin M. Bioelectrical control of positional information in development and regeneration: a review of conceptual and computational advances. Prog Biophys Mol Biol. 2018;137:52-68.

7. Weiß I, Bohrmann J. Electrochemical patterns during Drosophila oogenesis: ion-transport mechanisms generate stage-specific gradients of $\mathrm{pH}$ and membrane potential in the follicle-cell epithelium. BMC Dev Biol. 2019;19:12.

8. Weiß I, Bohrmann J. Electrochemical gradients are involved in regulating cytoskeletal patterns during epithelial morphogenesis in the Drosophila ovary. BMC Dev Biol. 2019;19:22.

9. González-Reyes A, Elliott H, St Johnston D. Polarization of both major body axes in Drosophila by gurken-torpedo signalling. Nature. 1995;375:654-8.

10. Roth S, Lynch JA. Symmetry breaking during Drosophila oogenesis. Cold Spring Harb Perspect Biol. 2009;1:a001891.
11. Spradling AC. Developmental genetics of oogenesis. In: Bate M, Martinez Arias A, editors. The development of Drosophila melanogaster. Cold Spring Harbor, New York: Cold Spring Harbor Laboratory Press; 1993. p. 1-70.

12. Cáceres L, Nilson LA. Production of gurken in the nurse cells is sufficient for axis determination in the Drosophila oocyte. Development. 2005;132:234553.

13. Duchek P, Rørth P. Guidance of cell migration by EGF receptor signaling during Drosophila oogenesis. Science. 2001;291:131-3.

14. Neuman-Silberberg FS, Schüpbach T. The Drosophila dorsoventral patterning gene gurken produces a dorsally localized RNA and encodes a TGF alpha-like protein. Cell. 1993;75:165-74.

15. Neuman-Silberberg FS, Schüpbach T. The Drosophila TGF-alpha-like protein Gurken: expression and cellular localization during Drosophila oogenesis. Mech Develop. 1996;59:105-13.

16. Nilson LA, Schüpbach T. EGF receptor signaling in Drosophila oogenesis. Curr Top Dev Biol. 1999:44:203-43.

17. Roth S, Neuman-Silberberg FS, Barcelo G, Schüpbach T. cornichon and the EGF receptor signaling process are necessary for both anterior-posterior and dorsal-ventral pattern formation in Drosophila. Cell. 1995:81:967-78.

18. González-Reyes A, St Johnston D. Patterning of the follicle cell epithelium along the anterior-posterior axis during Drosophila oogenesis. Development. 1998;125:2837-46

19. Zhao T, Graham OS, Raposo A, St Johnston D. Growing microtubules push the oocyte nucleus to polarize the Drosophila dorsal-ventral axis. Science. 2012;336:999-1003.

20. van Eeden F, St Johnston D. The polarisation of the anterior-posterior and dorsal-ventral axes during Drosophila oogenesis. Curr Opin Genet Dev. 1999; 9:396-404.

21. Guichet A, Peri F, Roth S. Stable anterior anchoring of the oocyte nucleus is required to establish dorsoventral polarity of the Drosophila egg. Dev Biol. 2001;237:93-106.

22. Levin $\mathrm{M}$, Thorlin $\mathrm{T}$, Robinson $\mathrm{KR}$, Nogi T, Mercola M. Asymmetries in $\mathrm{H}^{+} / \mathrm{K}^{+}$ATPase and cell membrane potentials comprise a very early step in leftright patterning. Cell. 2002;111:77-89.

23. Aw S, Koster JC, Pearson W, Nichols CG, Shi NQ, Carneiro K, Levin M. The ATPsensitive $\mathrm{K}(+)$-channel (K (ATP)) controls early left-right patterning in Xenopus and chick embryos. Dev Biol. 2010;346:39-53.

24. Pai VP, Aw S, Shomrat T, Lemire JM, Levin M. Transmembrane voltage potential controls embryonic eye patterning in Xenopus laevis. Development. 2012;139:313-23.

25. Beane WS, Morokuma J, Adams DS, Levin M. A chemical genetics approach reveals $\mathrm{H}, \mathrm{K}$-ATPase-mediated membrane voltage is required for planarian head regeneration. Chem Biol. 2011;18:77-89.

26. Beane WS, Morokuma J, Lemire JM, Levin M. Bioelectric signaling regulates head and organ size during planarian regeneration. Development. 2013;140: 313-22.

27. Busa WB. Mechanisms and consequences of $\mathrm{pH}$-mediated cell regulation. Annu Rev Physiol. 1986;48:389-402.

28. Schmoller KM, Köhler S, Crevenna AH, Wedlich-Söldner R, Bausch AR. Modulation of cross-linked actin networks by pH. Soft Matter. 2012;8:9685.

29. Köhler S, Schmoller KM, Crevenna AH, Bausch AR. Regulating contractility of the actomyosin cytoskeleton by pH. Cell Rep. 2012;2:433-9.

30. Priel A, Ramos AJ, Tuszynski JA, Cantiello HF. A biopolymer transistor: electrical amplification by microtubules. Biophys J. 2006;90:4639-43.

31. Cantero MDR, Perez PL, Smoler M, Villa Etchegoyen C, Cantiello HF. Electrical oscillations in two-dimensional microtubular structures. Sci Rep. 2016:6:27143.

32. Chifflet S, Hernández JA, Grasso S, Cirillo A. Nonspecific depolarization of the plasma membrane potential induces cytoskeletal modifications of bovine corneal endothelial cells in culture. Exp Cell Res. 2003;282:1-13.

33. Chifflet S, Hernández JA. The plasma membrane potential and the organization of the actin cytoskeleton of epithelial cells. Int J Cell Biol. 2012; 2012:121424.

34. Bohrmann J, Dorn A, Sander K, Gutzeit H. The extracellular electrical current pattern and its variability in vitellogenic Drosophila follicles. J Cell Sci. 1986; 81:189-206.

35. Bohrmann J, Huebner E, Sander K, Gutzeit H. Intracellular electrical potential measurements in Drosophila follicles. J Cell Sci. 1986;81:207-21.

36. Bohrmann J, Frey A, Gutzeit HO. Observations on the polarity of mutant Drosophila follicles lacking the oocyte. Roux's Arch Dev Biol. 1992;201: 268-74. 
37. Gutzeit HO. The role of microtubules in the differentiation of ovarian follicles during vitellogenesis in Drosophila. Roux's Arch Dev Biol. 1986;195: 173-81.

38. Gutzeit HO. The microfilament pattern in the somatic follicle cells of midvitellogenic ovarian follicles of Drosophila. Eur J Cell Biol. 1990;53:349-56.

39. Gutzeit HO, Eberhardt W, Gratwohl E. Laminin and basement membraneassociated microfilaments in wild-type and mutant Drosophila ovarian follicles. J Cell Sci. 1991;100:781-8.

40. Bohrmann J, Braun B. Na, K-ATPase and V-ATPase in ovarian follicles of Drosophila melanogaster. Biol Cell. 1999;91:85-98.

41. Bohrmann J, Haas-Assenbaum A. Gap junctions in ovarian follicles of Drosophila melanogaster: inhibition and promotion of dye-coupling between oocyte and follicle cells. Cell Tissue Res. 1993;273:163-73.

42. Bohrmann J, Zimmermann J. Gap junctions in the ovary of Drosophila melanogaster: localization of innexins 1, 2, 3 and 4 and evidence for intercellular communication via innexin-2 containing channels. BMC Dev Biol. 2008:8:111.

43. Lautemann J, Bohrmann J. Relating proton pumps with gap junctions: Colocalization of ductin, the channel-forming subunit c of V-ATPase, with subunit a and with innexins 2 and 3 during Drosophila oogenesis. BMC Dev Biol. 2016;16:24.

44. Mathews J, Levin M. Gap junctional signaling in pattern regulation: physiological network connectivity instructs growth and form. Dev Neurobiol. 2017;77:643-73.

45. Neuman-Silberberg FS, Schüpbach T. Dorsoventral axis formation in Drosophila depends on the correct dosage of the gene gurken. Development. 1994;120:2457-63.

46. Thio GL, Ray RP, Barcelo G, Schüpbach T. Localization of gurken RNA in Drosophila oogenesis requires elements in the $5^{\prime}$ and $3^{\prime}$ regions of the transcript. Dev Biol. 2000;221:435-46.

47. Robb JA. Maintenance of imaginal discs of Drosophila melanogaster in chemically defined media. J Cell Biol. 1969;41:876-85.

48. Bohrmann J. In vitro culture of Drosophila ovarian follicles: the influence of different media on development, RNA synthesis, protein synthesis and potassium uptake. Roux's Arch Dev Biol. 1991;199:315-26.

49. King RC. Ovarian development in Drosophila melanogaster. New York: Academic Press; 1970

\section{Publisher's Note}

Springer Nature remains neutral with regard to jurisdictional claims in published maps and institutional affiliations.

Ready to submit your research? Choose BMC and benefit from:

- fast, convenient online submission

- thorough peer review by experienced researchers in your field

- rapid publication on acceptance

- support for research data, including large and complex data types

- gold Open Access which fosters wider collaboration and increased citations

- maximum visibility for your research: over $100 \mathrm{M}$ website views per year

At $\mathrm{BMC}$, research is always in progress.

Learn more biomedcentral.com/submissions 